\title{
Wall-shear stress fluctuations in a supersonic turbulent boundary layer over an expansion corner
}

\section{Fulin Tong , Jianqiang Chen , Dong Sun \& Xinliang Li}

To cite this article: Fulin Tong, Jianqiang Chen, Dong Sun \& Xinliang Li (2020): Wall-shear stress fluctuations in a supersonic turbulent boundary layer over an expansion corner, Journal of Turbulence, DOI: $10.1080 / 14685248.2020 .1797058$

To link to this article: https://doi.org/10.1080/14685248.2020.1797058

\section{曲 Published online: 23 Jul 2020.}

Submit your article to this journal $\widetilde{ }$

Џ Article views: 13

Q View related articles $₫$

View Crossmark data $₫$ 


\title{
Wall-shear stress fluctuations in a supersonic turbulent boundary layer over an expansion corner
}

\author{
Fulin Tong $^{a, b, c}$, Jianqiang Chen ${ }^{b, c}$, Dong Sun ${ }^{b, c}$ and Xinliang Li $\mathbb{D}^{a, d}$ \\ a LHD, Institute of Mechanics, Chinese Academy of Sciences, Beijing, People's Republic of China; ${ }^{\text {b State Key }}$ \\ Laboratory of Aerodynamics, China Aerodynamics Research and Development Center, Mianyang, People's \\ Republic of China; ' ${ }^{C}$ Computational Aerodynamics Institute, China Aerodynamics Research \& Development \\ Center, Mianyang, People's Republic of China; ${ }^{\mathrm{d} S}$ School of Engineering Science, University of Chinese \\ Academy of Sciences, Beijing, People's Republic of China
}

\begin{abstract}
The effect of wall expansion on the structural and statistical characteristics of wall-shear stress (WSS) fluctuations was investigated by direct numerical simulations of a supersonic turbulent boundary layer over a sharp expansion corner with various deflection angles $\left(\beta=0^{0}, 2^{0}, 5^{0}\right.$ and $\left.10^{\circ}\right)$. It is found that the two-dimensional fields of WSS are characterised as streamwise-elongated streaky structures being aligned in the spanwise direction, resembling low- and highspeed streaks in the buffer region of the flow. Due to the relaminarization effect, these WSS steaks experience a sudden weakening shortly after the expansion corner, but present gradual regrowth with their length scales even exceeding those of the flat-plate case in the far downstream. A strong streamwise-alignment of the instantaneous WSS vector is evident in the case of the largest deflection angle, suggesting a distinct reduction of the intermittency in the relaminarization process. Furthermore, the characteristic time scale of the spanwise component of WSS is quasi-invariant to the expansion effect, while the peak frequency of the streamwise component increases with the increase of the deflection angle.
\end{abstract}

\section{ARTICLE HISTORY}

Received 20 March 2020

Accepted 2 July 2020

\section{KEYWORDS}

Wall-shear stress; expansion corner; supersonic turbulent boundary layer; direct numerical simulation

\section{Introduction}

Supersonic turbulent boundary layer (TBL) passing through an expansion corner is a baseline prototype of various supersonic engineering applications [1]. It has been known that turbulence in such a kind of flow is greatly affected by the combination of favour pressure gradient, streamline curvature and bulk dilatation. All these factors will jointly lead to the so-called relaminarization process [2], which describes a significant reduction of turbulence intensities when accelerating flow passes through the expansion corner. The kinematics and dynamics involved in this relaminarization process are rather complex, and the underling physical mechanisms haven not been fully understood yet.

CONTACT Fulin Tong 515363491@qq.com E LHD, Institute of Mechanics, Chinese Academy of Sciences, Beijing 100190, People's Republic of China; State Key Laboratory of Aerodynamics, China Aerodynamics Research and Development Center, Mianyang 621000, People's Republic of China; Computational Aerodynamics Institute, China Aerodynamics Research \& Development Center, Mianyang 621000, People's Republic of China 
To date back, Dussauge \& Gaviglio [3] might be the first to notice that the evolution of the Reynolds stress was dominated by the production rate of the bulk dilation. Smith \& Smits [4] observed that the mass flux fluctuation presented only a slight change after the expansion corner, in contrast to the dramatic decrease of the velocity fluctuation. Arnette et al. $[5,6]$ experimentally investigated the influence of expansion level on turbulence structures in a compressible boundary layer. They observed that small-scale turbulent structures in the near-wall region were significantly quenched, while large-scale ones in the outer region were less affected. They further concluded that the bulk dilatation was the primary factor for the stabilisation of turbulent flow. The following experiments $[7,8]$ mainly focused on the characteristics of pressure fluctuations at the wall. It was shown that wall pressure fluctuations after the expansion corner still follow a Gaussian distribution, similar to that in a flat-plate TBL; nevertheless, the spreading of the distribution was dramatically attenuated. Furthermore, the reduction levels of low-frequency (large-scale) components in the spectra of wall pressure fluctuations were seen to be comparably smaller than those of high-frequency (small-scale) ones. This suggests that small-scale fluctuations are more sensitive to the expansion effect.

In recent days, large-eddy simulations (LESs) and direct numerical simulations (DNSs) of this kind of flow provide multi-dimensional information that are difficult to be accessed by experimental methods. For example, Nguyen et al. [9] numerically studied the rapid distortion and relaxation of near-wall turbulence in the expansion region. Termmoto et al. [10] performed LES to study a supersonic TBL with $R e_{\theta}=5000$ over an expansion corner of $12^{\circ}$ with a focus on the dilatation effect. They found that the bulk dilatation and the non-equilibrium velocity profile played an equivalent role in the accelerating process. Sun et al. [11] recently investigated the recovery of a supersonic TBL over an expansion corner at Mach number 2.7 and deflection angle of $2^{0}$ and $4^{0}$ via DNS. A characteristic two-layer structure was proposed to explain the different recovery behaviours between the inner layer and outer layer.

Despite the large bulk of existing studies, the knowledge on the kinematics of a supersonic TBL subjected to the expansion effect is still incomplete. One of the important issues that have received less attention in the past is the modulation of wall-shear stress (WSS) fluctuations, which is of great importance for engineering applications. In the fundamental side, WSS is an ideal indicator to characterise both the relaminarization process and the following regrowth of turbulent fluctuations. It might also contribute to unveiling the underlying physical mechanisms of the expansion effect. Based on this consideration, a fully developed supersonic TBL at Mach number 2.9 subjected to an expansion corner with various deflection angles are studied via DNS. Here, we mainly focus on the effect of deflection angle on both the statistics of WSS fluctuations and their spatial structures. It is hoped that the present study will provide new insights on near-wall kinematics of a compressible TBL that are affected by the expansion effect.

\section{Numerical Methodology}

(A) Flow configuration

A sketch of the computational domain scaled by the unit $\mathrm{mm}$ is shown in Figure 1, where the inflow is from the left to the right. The origin of the coordinate system is set 


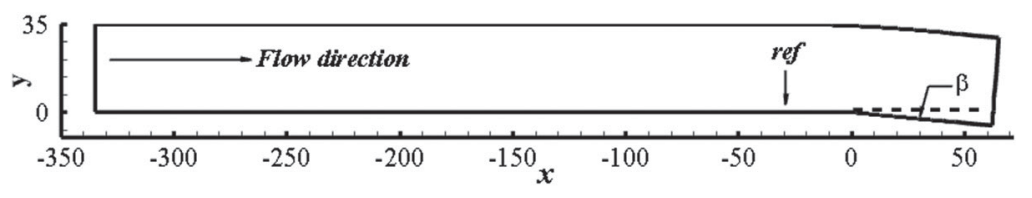

Figure 1. A sketch of the computational domain in the $x-y$ plane used for the simulations.

Table 1. Conditions for the incoming TBL at $x_{\text {ref }}$.

\begin{tabular}{ccccccc}
\hline $\mathrm{M}_{\infty}$ & $T_{\infty}(\mathrm{K})$ & $\mathrm{U}_{\infty}(\mathrm{m} / \mathrm{s})$ & $\delta_{\text {ref }}(\mathrm{mm})$ & $\delta^{*}(\mathrm{~mm})$ & $\theta(\mathrm{mm})$ & $\mathrm{Re}_{\theta}$ \\
\hline 2.9 & 108.4 & 604.0 & 6.5 & 2.06 & 0.41 & 2300 \\
\hline
\end{tabular}

at the tip of the expansion corner. In the present study, four expansion angles, i.e. $\beta=0^{0}$ (flat-plate), $2^{0}, 5^{0}$ and $10^{0}$ are considered. The overall size of the domain is $L_{x} \times L_{y} \times$ $L_{z}=395 \times 35 \times 14$, with $L_{x}, L_{y}$ and $L_{z}$ being the length in the streamwise, wall-normal and spanwise directions, respectively. The $x$ station at 30 upstream of the origin, i.e. $x_{\text {ref }}=-30$, is selected as the reference station, at which the local viscous length $\left(l_{\text {ref }}^{*}\right)$ and the boundary layer thickness $\left(\delta_{\text {ref }}\right)$ is used as the global inner and outer length scale, respectively.

The free-stream parameters are given in Table 1, including the Mach number $M_{\infty}$, static temperature $T_{\infty}$ and the thickness of turbulent boundary layer at the reference station, which are in good accordance with the Mach 2.9 experiments of Bookey et al. [12] and the previous DNS simulation of Wu et al. [13]. The nominal thickness $\delta_{\text {ref }}$ of turbulent boundary layer based on the $99 \%$ inflow velocity $U_{\infty}$ is estimated to be $6.5 \mathrm{~mm}$. The corresponding momentum thickness $\theta$ and the Reynolds number $R e_{\theta}$ are $0.41 \mathrm{~mm}$ and 2300, respectively.

A total of four DNS cases are performed in our simulation, including the zero-pressure gradient flat-plate turbulent boundary layer without expansion corner $\left(\beta=0^{0}\right)$ and the other three cases of expansion corner with varying deflection angles $\left(\beta=2^{0}, 5^{0}\right.$ and $\left.10^{0}\right)$. As suggested by Narasimha \& Viswanath [14], the relaminarization of near-wall turbulence occurs for $\Delta p / \tau_{0}>70$, with $\Delta p$ and $\tau_{0}$ being the pressure drop across the corner and the WSS just upstream the corner $(x=0)$, respectively. For the present studied expansion angle of $\beta=2^{0}, 5^{0}$ and $10^{\circ}, \Delta p / \tau_{0}$ is about 34,45 and 77 , respectively. Therefore, it can be expected that near-wall turbulence would be dramatically suppressed with $\beta=10^{\circ}$.

B. Numerical methods and boundary conditions

The governing equations are the compressible conservative Navier-Stokes equations in the curvilinear coordinate, which are defined as

$$
\partial_{t} U+\partial_{\xi}\left(F+F_{v}\right)+\partial_{\eta}\left(G+G_{v}\right)+\partial_{\zeta}\left(H+H_{v}\right)=0
$$

Here, the vector $U=J^{-1}(\rho, \rho u, \rho v, \rho w, \rho E)$ denotes the conservative vector flux, consisting of the density $\rho$, the corresponding velocity $(u, v, w)$ in the three directions and the total energy $\rho E$. The variable $J^{-1}$ is the Jacobin in the transformation from the Cartesian coordinate $(x, y, z)$ to the curvilinear coordinate $(\xi, \eta, \zeta)$. The vectors $F$ and $F_{v}$ are the 
corresponding convective and viscous flux terms in the direction $\xi$, written as

$$
F=J^{-1}\left[\begin{array}{c}
\rho \tilde{U} \\
\rho u \tilde{U}+\xi_{x} p \\
\rho v \tilde{U}+\xi_{y} p \\
\rho w \tilde{U}+\xi_{z} p \\
(\rho E+p) \tilde{U}
\end{array}\right] \text { and } F_{v}=J^{-1}\left[\begin{array}{c}
0 \\
\xi_{x} \sigma_{11}+\xi_{y} \sigma_{21}+\xi_{z} \sigma_{31} \\
\xi_{x} \sigma_{12}+\xi_{y} \sigma_{22}+\xi_{z} \sigma_{32} \\
\xi_{x} \sigma_{13}+\xi_{y} \sigma_{23}+\xi_{z} \sigma_{33} \\
\xi_{x} S_{1}+\xi_{y} S_{2}+\xi_{z} S_{3}
\end{array}\right]
$$

where

$$
\begin{gathered}
\tilde{U}=u \xi_{x}+v \xi_{y}+w \xi_{z}, \\
S_{1}=u \sigma_{11}+v \sigma_{21}+w \sigma_{31}-q_{1}, \\
S_{2}=u \sigma_{12}+v \sigma_{22}+w \sigma_{32}-q_{2}, \\
S_{3}=u \sigma_{13}+v \sigma_{23}+w \sigma_{33}-q_{3}
\end{gathered}
$$

and

$$
\begin{aligned}
\sigma_{i j} & =2 \mu \frac{\partial u_{i}}{\partial x_{j}}-\frac{2}{3} \mu \frac{\partial u_{k}}{\partial x_{k}} \delta_{i j}, \\
q_{i} & =-k \frac{\partial T}{\partial x_{i}} .
\end{aligned}
$$

The perfect gas is chosen as the working air and the relationship between the pressure $p$ and the temperature $T$ is given by the ideal gas law. The Sutherland law is used to obtain the viscosity $\mu$. For clarity, the convective and viscous flux terms in the other two directions $(\eta$ and $\zeta$ ) are not given.

An in-house code, i.e. Opencfd-SC, was used to perform DNS calculation. This code has been successfully applied to a series of studies involving shock waves and compressible boundary layers. Details about the numerical algorithms and the code validation can be referred to Tong et al. [15]. Here, the numerical algorithms of the solver are introduced briefly. The convective terms are discretised by a bandwidth-optimised fourthorder weighted essentially non-oscillatory (WENO) scheme [16] with the Steger-Warming vector flux splitting method. To reduce the numerical dissipation, the linear part of original WENO method is optimised. An eighth-order accuracy central difference scheme is used to calculate the viscous flux terms and the time integration is performed using the third-order explicit Runge-Kutta method.

The boundary layer conditions are described as follows. A steady laminar velocity profile is imposed at the inlet of the computation domain and a non-reflecting boundary condition with a sponge layer is used at the outlet. A no-slip and isothermal boundary condition $\left(T_{w}=307 \mathrm{~K}\right)$ is applied at the bottom wall, while a non-reflecting condition on the upper boundary and a periodic condition in the spanwise direction. To generate a fully developed supersonic TBL before the expansion corner, a band of tripping disturbance was imposed at the wall ranging from $x=-305$ to $x=-285$. The tripping disturbance was composed of multi-frequency wall-normal blowing and suction, whose amplitude and frequencies are identical to those used in Tong et al. [15]. 


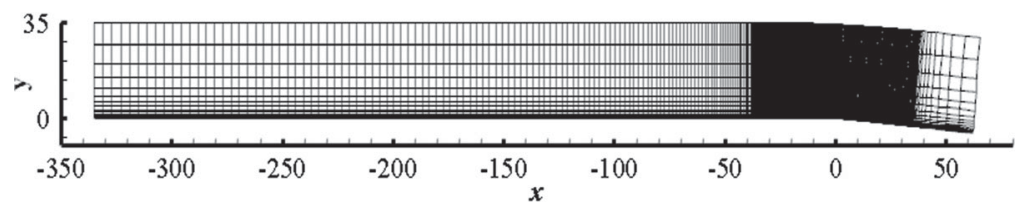

Figure 2. A sketch of the computational grid. The grid is plotted at intervals of every ten grid points in both $x$ and $y$ directions.

Table 2. Grid parameters for the grid-sensitivity study.

\begin{tabular}{lccc}
\hline Case & $\mathrm{L}_{x} \times \mathrm{L}_{y} \times \mathrm{L}_{z}$ & $\mathrm{~N}_{x} \times \mathrm{N}_{y} \times \mathrm{N}_{z}$ & $\Delta x^{+} \times\left(\Delta y_{w}{ }^{+}-\Delta y_{e}^{+}\right) \times \Delta z^{+}$ \\
\hline Grid A & $395 \times 35 \times 14$ & $3200 \times 240 \times 280$ & $1.88 \times(0.47-5.64) \times 3.31$ \\
Grid B & $395 \times 35 \times 14$ & $4500 \times 240 \times 280$ & $1.11 \times(0.47-5.64) \times 3.31$ \\
Grid C & $395 \times 35 \times 14$ & $3200 \times 320 \times 280$ & $1.88 \times(0.47-4.23) \times 3.31$ \\
Grid D & $395 \times 35 \times 14$ & $3200 \times 240 \times 560$ & $1.88 \times(0.47-5.64) \times 1.65$ \\
Sun et al. [11] & $185 \times 26 \times 15$ & $2305 \times 241 \times 289$ & $6.5 \times(0.8-9.8) \times 4.1$ \\
\hline
\end{tabular}

\section{Grid and numerical validations}

A sketch of the computational grid is shown in Figure 2 and the details of grid-sensitivity are listed in Table 2. We use a grid consisting of $3200 \times 240 \times 280$ (Grid A) points to discretise the computational domain along the streamwise, wall-normal and spanwise directions, respectively, where the mesh lines are perpendicular to the wall. The grid points in the expansion corner are equally spaced in the streamwise direction from $x=-35$ to $x=35$, and a sponge region with gradually coarsened grid is placed at $x>35$ to eliminate the disturbances from the outlet. Unless otherwise stated, the plus notation denotes the inner scaling based on the wall unit at the reference station $x_{\text {ref }}$. In the wall-normal direction, the points of grid are clustered toward the wall and exponentially stretched outward to ensure that there are 150 nodes distributed within the boundary layer. The grid spacing above the wall and at the edge of boundary layer is $\Delta y_{w}^{+}=0.47$ and $\Delta y_{e}^{+}=5.64$, respectively, where the superscript $w$ and $e$ denote the wall parameter and the edge of boundary layer. In the spanwise direction, the grid points are equally distributed with uniform spacing $\Delta z^{+}=3.31$. The grid resolution in the expansion section of the present study is comparable to that of the DNS of Sun et al. [11]. The total amount of the grid is about 200 million, while the calculation takes about $3.07 \times 10^{5} \mathrm{CPU} /$ hours for each case.

To validate the grid resolution, a grid sensitivity study, listed in Table 2, is performed by refining the grid points in the streamwise, wall-normal and spanwise directions, respectively. As shown in Figure 3, both the wall pressure and skin-friction show no significant changes, varying less than $6 \%$. It is confirmed that the resolution of Grid A used in the present simulation is sufficiently fine to ensure the convergence. The two-point spanwise correlation is also validated to check the domain size in the spanwise direction, which is defined as [17]

$$
R_{\alpha \alpha}\left(r_{z}\right)=\sum_{k=1}^{N z-1} \alpha_{k} \alpha_{k+k_{r}}, \quad k_{r}=0,1, \ldots, k-1
$$




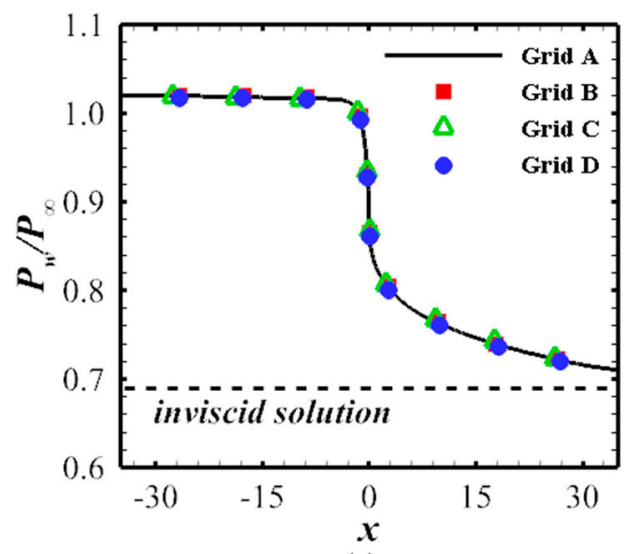

(a)

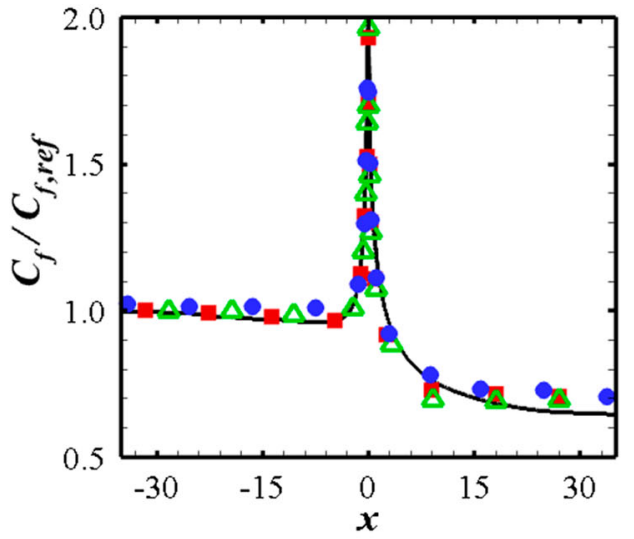

(b)

Figure 3. Grid-sensitivity study: distributions of the wall pressure (a) and skin-friction (b) for $\beta=5^{0}$.

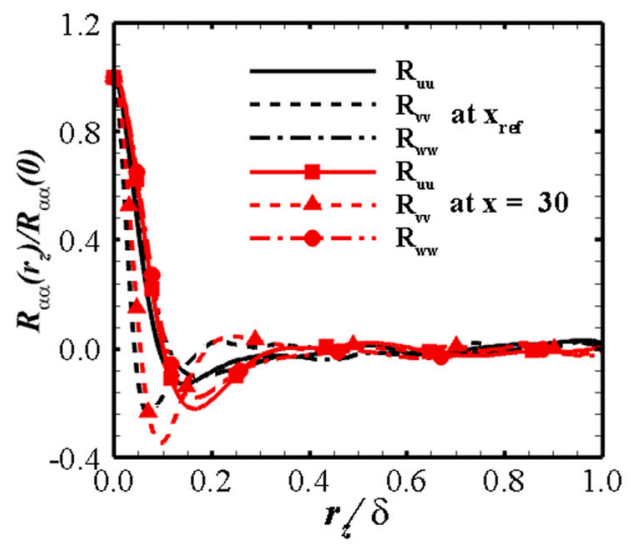

(a)

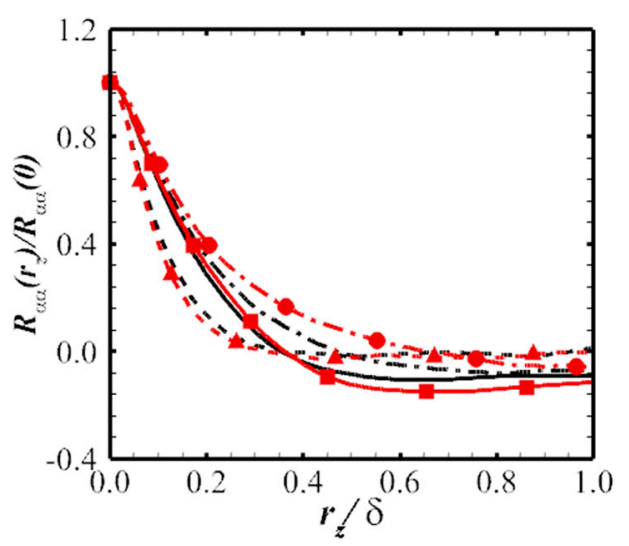

(b)

Figure 4. Distributions of the two-point autocorrelation as a function of spanwise space: (a) $y^{+}=10$; (b) $y / \delta_{\text {ref }}=0.6$.

where $\alpha$ represents the fluctuating velocity and $r_{\mathrm{z}}$ denotes the spanwise coordinate $k_{r} \Delta \mathrm{z}$. Figure $4(\mathrm{a}, \mathrm{b})$ show the spanwise distribution of the fluctuating velocity correlations at various wall-normal locations, respectively. All profiles at $x_{\text {ref }}$ and $x=30$ decrease rapidly toward zero within the half of domain width, suggesting that the spanwise span $\left(L_{z}\right)$ of this simulation (about twice of $\delta_{\text {ref }}$ ) is sufficiently large to encompass large-scale structures in the outer region of the TBL.

The statistical steadiness is obtained after a time interval $T_{0} \mathrm{U}_{\infty} / \delta_{\text {in }}=400$, where $\delta_{\text {in }}$ is the boundary layer thickness at the domain inlet. As has been stated in the above, the present study mainly focuses on the characteristics of WSS. In this sense, 10,000 snapshots of instantaneous WSS distribution, i.e. $\vec{\tau}(x, z)$ with $\vec{\tau}=\left(\tau_{x}, \tau_{z}\right)$, are collected over a time period of $\Delta T_{0} \mathrm{U}_{\infty} / \delta_{\text {in }}=800$ to ensure the statistical convergence. Note that $\vec{\tau}$ is estimated by the wall-normal velocity gradient at the wall. Both the streamwise and spanwise components are considered here. Note that the fluctuation of general variable is calculated 


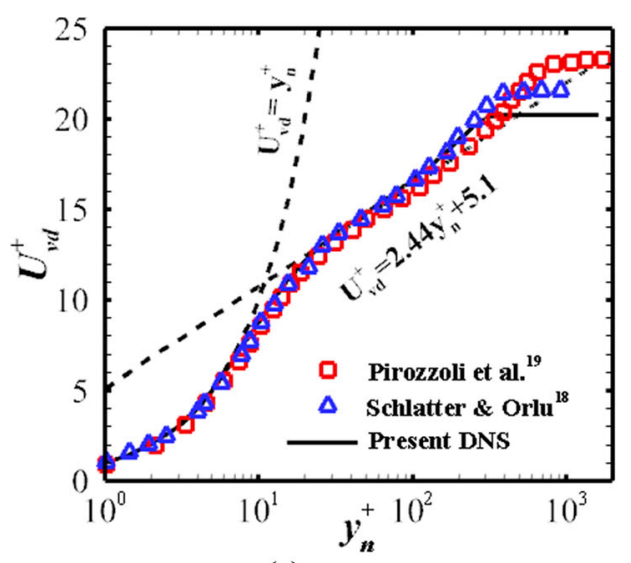

(a)

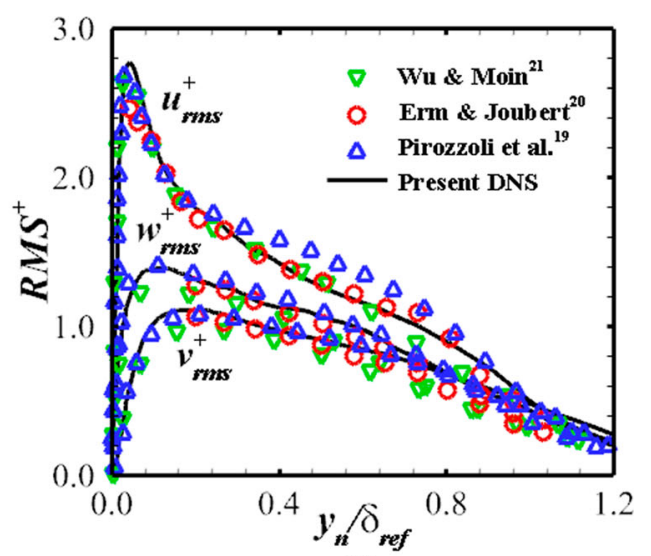

(b)

Figure 5. Turbulence statistics at the reference station $x_{\text {ref: }}$ (a) van Driest-transformed mean velocity profile; (b) turbulence intensities in outer scaling.

using either the Reynolds decomposition $f^{\prime}=f-\bar{f}$ or the Favre decomposition $f^{\prime}=f-\widetilde{f}$ where $\bar{f}$ denotes the average in time and the spanwise direction and $\tilde{f}$ is correspond to the density-weighted average defined as $\tilde{f}=\overline{\rho f} / \bar{\rho}$.

\section{Results and discussion}

(A) Incoming turbulent boundary layer

The turbulence statistics of the incoming TBL taken at the reference location are reported in Figure 4, together with those in both previous DNS $[18,19]$ and experiments [20]. In Figure 5(a), the profile of the van-Direst transferred mean velocity $U_{\mathrm{vd}}{ }^{+}$is present with the incompressible DNS data of Schlatter \& Orlu [18] and compressible numerical results of Pirozzoli et al. [19], where $U_{\mathrm{vd}}{ }^{+}$is defined as $U_{\mathrm{vd}}^{+}=\int_{0}^{\overline{U_{e}}}\left(\bar{\rho} / \overline{\rho_{w}}\right)^{1 / 2} \mathrm{du}$ with $\overline{U_{e}}$ being the mean streamwise velocity at the edge of turbulent boundary layer. Clearly, the comparison is satisfactory that the linear law is attained for $y_{n}{ }^{+}<7$ and the region for $40<y_{n}{ }^{+}<100$ is characterised by the logarithmic scaling. Figure 5(b) shows the distribution of the root mean square (RMS + ) of velocity fluctuations scaled by $\sqrt{\bar{\rho} / \overline{\rho_{w}}} / u_{\tau}$ as a function of the wall-normal distance $y_{\mathrm{n}}$ in outer scaling. It is seen that the turbulence intensities match well with the compressible flow data of Pirozzoli et al. [19] that the streamwise velocity fluctuation attains a peak value of 2.7 at $y_{\mathrm{n}} / \delta_{\text {ref }}=0.045$. As expected, all the density-scaled profiles are in good agreement with the low-speed experimental data of Erm \& Jouber [20] and the incompressible DNS data of Wu \& Moin [21].

The pre-multiplied energy spectra of the fluctuating WSS at the reference location is also evaluated in Figure 6 as a function of the non-dimensional angular frequency $\omega^{+}=\omega v_{w} / u_{\tau}^{2}$, where the spectra is normalised by the mean streamwsie WSS $\left(\tau_{x, \text { av }}\right)$. The published DNS data of the incompressible channel flow of Hu et al. [22] and the flat-plate turbulent boundary layer of Daniel et al. [23] is also included in this figure. The spectra are computed using the Welch's power spectral density estimate and averaged in the spanwise direction. It can be clearly seen that the present DNS results match well with the 


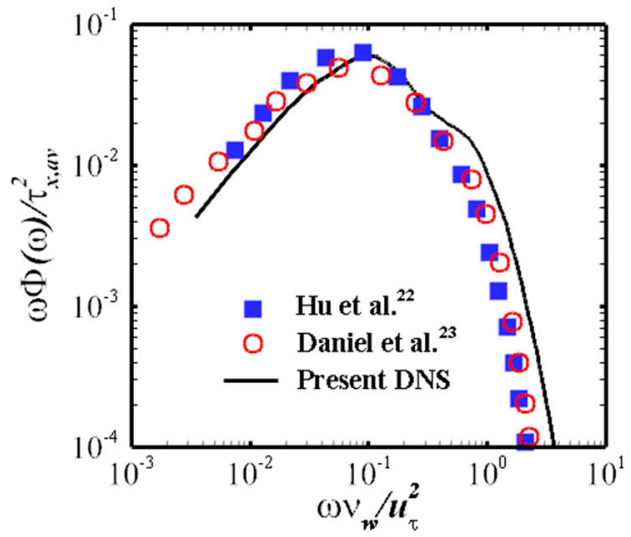

(a)

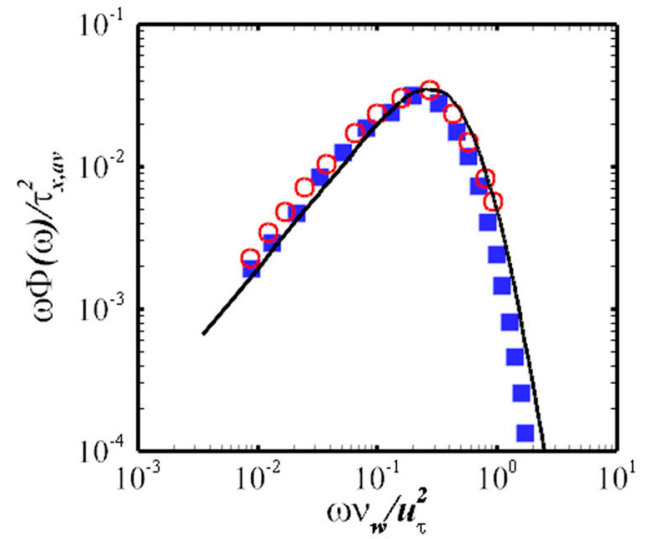

(b)

Figure 6. Pre-multiplied energy spectra of the wall shear stress fluctuations at the reference location $x_{\text {ref }}$ : (a) $\tau_{x^{\prime}}^{\prime}$ (b) $\tau_{z^{\prime}}^{\prime}$.

incompressible data when using the inner scaling. The peak frequency of the streamwise component occurs at $\omega^{+}=0.1$, much smaller than that of the spanwise component attaining a maximum value at $\omega^{+}=0.26$. According to Daniel et al. [23], this is related to the fact that the energy-containing scale for the streamwise wall shear stress is much larger than that of the spanwise component.

Figure 7 shows the probability distribution function (PDF) of the fluctuating streamwise wall shear scaled by its r.m.s value, together with a Gaussian distribution for reference. Obviously, the present PDF profile exhibits a relatively skew behaviour and the peak value is attained nearly at $\tau_{x}^{\prime} / \tau_{x, \mathrm{rms}}=0.5$, in good agreement with the incompressible experimental data of duct flow by Grobe \& Schröder [24] at $R e_{\mathrm{H}}=15,000$ (Reynolds number based on the duct height), turbulent boundary layer by Nottebrock et al. [25] at $R e_{\theta}=4420$ and Channel flow by Sreenivasn \& Antonia [26] at $R e_{D}=6050$ (Reynolds number based on the channel half-height). It is further conformed that the PDF of the normalised streamwise wall shear stress fluctuations is independent of the Reynolds number, as reported in Grobe \& Schröder [24].

\section{B. Characteristics of spatial distribution}

Figure 8 illustrates typical snapshots of the normalised streamwise and spanwise components of the fluctuating WSS, i.e. $\tau_{x}^{\prime} / \tau_{x \text {,av }}$ and $\tau_{z}^{\prime} / \tau_{x \text {,av }}$, at various expansion angle $\beta$, respectively. Note that $\tau_{x \text {,av }}$ is the local mean WSS. Clearly, typical streak-like structures with alternating sign appear in both $\tau_{x}^{\prime}(x, z)$ and $\tau_{z}^{\prime}(x, z)$ in the upstream of the expansion corner, resembling those in both an incompressible turbulent duct flow [26] and an incompressible flat-plate TBL [27]. These streaky structures in WSS are attributed to near-wall streaks, whose spanwise meandering feature are inherited at the wall, as can be clearly seen in the wavy pattern of the $\tau_{z}^{\prime}$ component. Furthermore, the streamwise length scale of $\tau_{x}^{\prime}$ is much larger than that of $\tau_{z}^{\prime}$, while the wavy pattern of the former is less significant. Such a discrepancy is associated with the anisotropy of near-wall streaks [14]. More importantly, the spatial distributions are strongly affected by the expansion 


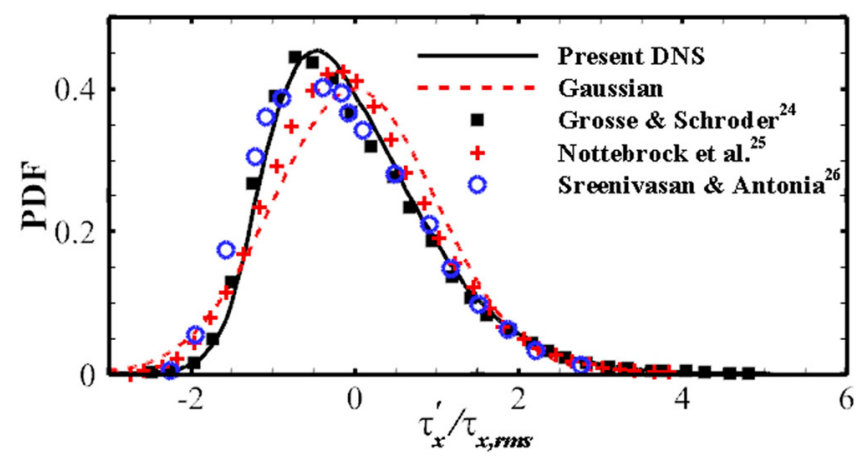

Figure 7. Probability distribution functions of the streamwise component of the fluctuating WSS.
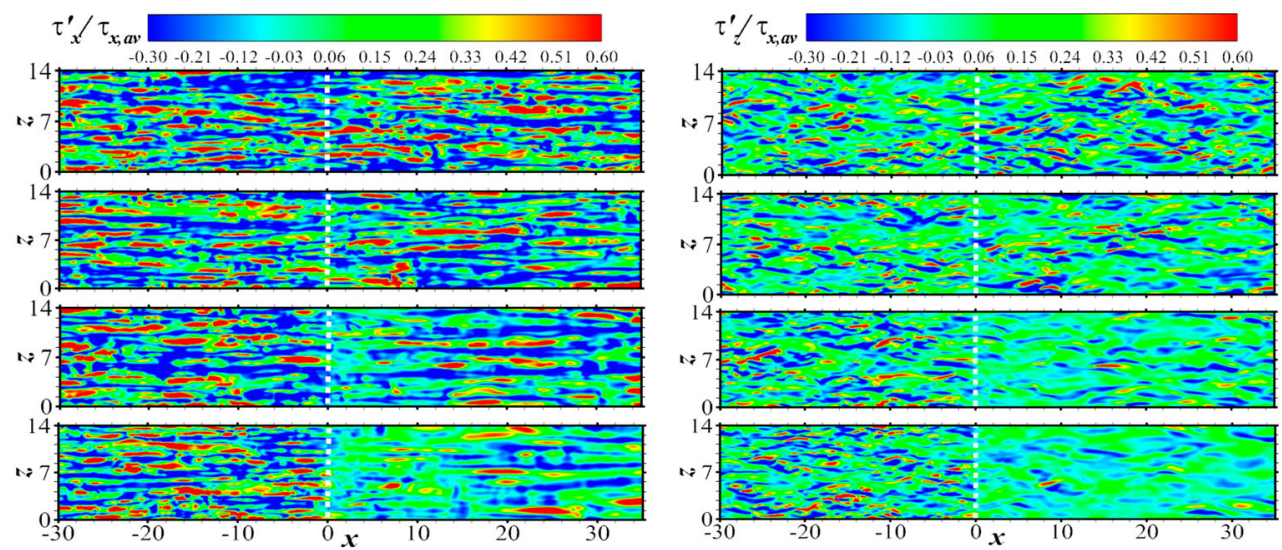

Figure 8. Contours of instantaneous streamwise (left panels) and spanwise (right panels) WSS fluctuation at various expansion angles, increasing from top to bottom $\left(\beta=0^{0}, 2^{0}, 5^{0}\right.$ and $\left.10^{0}\right)$. The white dashed lines denote the leading edge of the expansion corner.

angle, an increment of $\beta$ implying a weakness of the streaky structures. In particular, both the strength of WSS streaks and their population density present a sudden drop shortly after the expansion corner with $\beta=10^{\circ}$. This demonstrates the existence of the relaminarization process. However, a gradual recovery of these streaks are seen to occur in the downstream region (after $x=10$ ). When $x$ increases beyond 20, the length scales and strength of these structures become even larger than their counterparts in the upstream flat-plate section.

Such a regrowth phenomenon can be further quantitatively characterised by the map of two-point cross-correlation coefficient of WSS, i.e. $C_{\tau_{x}^{\prime} \tau_{x}^{\prime}}(\Delta x, \Delta z)$ and $C_{\tau_{z}^{\prime} \tau_{z}^{\prime}}(\Delta x, \Delta z)$, which is defined as

$$
C_{\phi^{\prime} \phi^{\prime}}\left(x_{0}+\Delta x, z_{0}+\Delta z\right)=\frac{\overline{\phi^{\prime}\left(x_{0}, z_{0}\right) \phi^{\prime}\left(x_{0}+\Delta x, z_{0}+\Delta z\right)}}{{\phi^{\prime 2}\left(x_{0}, z_{0}\right)}^{1 / 2}{\overline{\phi^{\prime}\left(x_{0}+\Delta x, z_{0}+\Delta z\right)}}^{1 / 2}},
$$

where $\phi^{\prime}$ represents the streamwise and spanwise WSS fluctuations, $\Delta x$ and $\Delta z$ denote the streamwise and spanwise separations, respectively. The probing point of the maps are set 


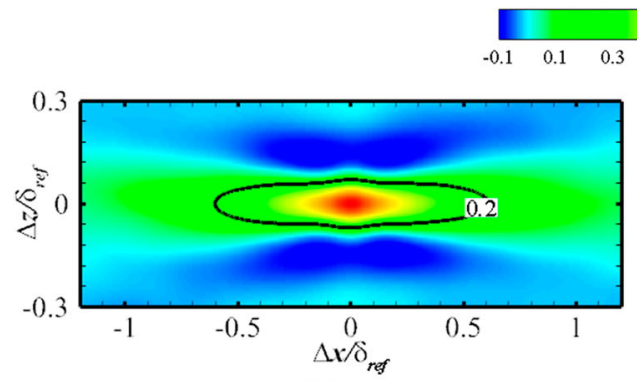

(a)

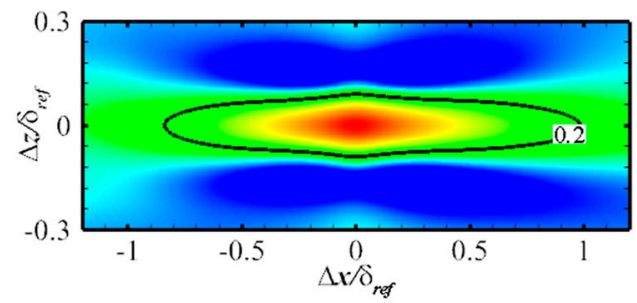

(c)

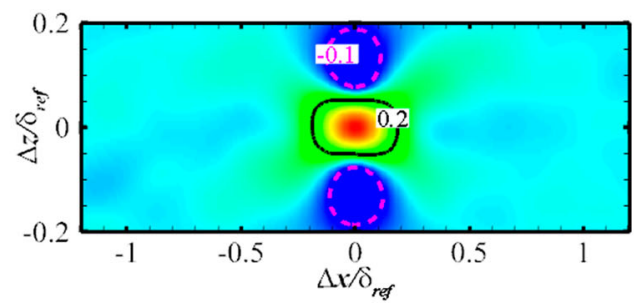

(b)

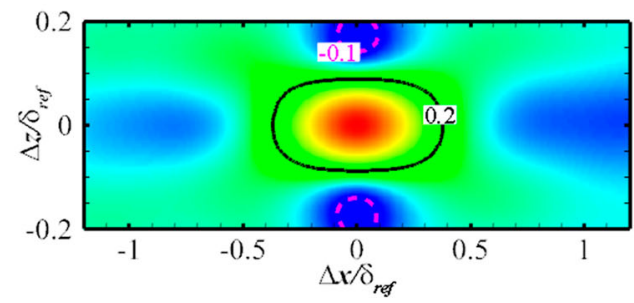

(d)

Figure 9. Maps of two-point correlation coefficient $C_{\boldsymbol{\tau}_{x^{\prime}} \boldsymbol{\tau}_{x}^{\prime}}$ (left panels) and $\boldsymbol{C}_{\boldsymbol{\tau}_{z^{\prime}}} \boldsymbol{\tau}_{z^{\prime}}$ (right panels) with the reference point at $x=20$ : $(\mathrm{a}, \mathrm{b}) \beta=0^{0}$; $(\mathrm{c}, \mathrm{d}) \beta=10^{0}$.

at $x_{0}=20$. The correlation results for $\beta=0^{0}$ and $\beta=10^{\circ}$ are shown in Figure 9, respectively. For the flat-plate, the iso-contours of both components are characterised by a typical feature of elliptical distribution for small spatial separations and become more elongated in the streamwise direction for large spatial distances, reflecting the increased anisotropic nature of the wall shear stress field. Relative to $\tau_{z}^{\prime}$, the correlation of $\tau_{x}^{\prime}$ has a significantly larger extent in the streamwise direction, supporting the observations in Figure 8. Apparently, significant differences can be observed in Figure $9(\mathrm{c}, \mathrm{d})$ to emphasise the expansion effect. In first place, a longer and wider statistical structure of both $\tau_{x}^{\prime}$ and $\tau_{z}^{\prime}$ is clearly evident for $\beta=10^{0}$, consistent with the visualisation in Figure 8. Secondly, a more-elliptical correlation shape is clearly identified, suggesting utterly different variations in both directions. Similar to Bernardini \& Pirozzoli [28], the integral length scales in the streamwise and spanwise directions, defined as

$$
\Lambda_{x}=\int^{C_{\Phi \Phi}}(\Delta x, 0) d \Delta x \text { and } \Lambda_{z}=\int^{C_{\Phi \Phi}}(0, \Delta z) d \Delta z
$$

are reported in Table 3. For $\tau_{x}^{\prime}$ and $\tau_{z}^{\prime}$, the streamwise integral length scale $\Lambda_{x}$ are consistently larger than the spanwise integral length scale $\Lambda_{z}$, supporting the elliptical shape of the contours. Notice that a clear dependency of $\Lambda_{x}$ and $\Lambda_{z}$ on the expansion effect is quantitatively established. As seen from Table 3, increasing the deflection angle $\beta$ leads to a steep increase in $\Lambda_{x}$, whereas $\Lambda_{z}$ is featured by a relatively slight increase. For instance, the values of $\Lambda_{x}$ and $\Lambda_{z}$ for $\beta=10^{0}$ are amplified by a factor of about 1.6 and 1.2, respectively, with respect to the flat-plate case. Considering that $\Lambda_{x}$ is much larger than $\Lambda_{z}$, it is believed that the differences in growth rate between the two directions are mainly responsible for the more-elliptical distributions. 
Table 3. Integral length scales $\Lambda_{x}$ and $\Lambda_{z}$ for the WSS fluctuations.

\begin{tabular}{lcccc}
\hline & \multicolumn{3}{c}{$\tau_{x}^{\prime}$} & $\tau_{z}^{\prime}$ \\
\cline { 2 - 5 } Case & $\Lambda_{x} / \delta_{\text {ref }}$ & $\Lambda_{z} / \delta_{\text {ref }}$ & $\Lambda_{x} / \delta_{\text {ref }}$ & $\Lambda_{z} / \delta_{\text {ref }}$ \\
\hline$\beta=0^{0}$ & 0.693 & 0.129 & 0.274 & 0.086 \\
$\beta=2^{0}$ & 0.826 & 0.142 & 0.340 & 0.087 \\
$\beta=5^{0}$ & 0.871 & 0.147 & 0.309 & 0.089 \\
$\beta=10^{0}$ & 1.120 & 0.158 & 0.568 & 0.133 \\
\hline
\end{tabular}

In order to understand the expansion effect on the structural characteristics of WSS fields, iso-surfaces of $Q$ criterion [29] and Lamb vector divergence [30] for $\beta=10^{0}$ are shown in Figure 10(a,b), respectively, to visualise the evolution of coherent vortex structures and momentum transports as the upstream TBL passes over the expansion corner. Notable differences are observed from the comparison between the upstream region and the expansion region. As shown in Figure 10(a), upstream the expansion corner, the hairpin-shaped vortices are mostly concentrated in the outer layer, whereas the near-wall region is populated by numerous elongated quasi-streamwise vortices. In the expansion region, although the small-scale vortices in the near-wall region are rapidly diminished and rarely observed, the large-scale vortices in the outer layer are relatively weakened and still visible with sparse distributions, consistent with the experimental observations of Arnette et al. [5,6]. A similar behaviour can be also found in Figure 10(b). In the upstream region, the momentum exchange inside the incoming TBL is characterised by the strong interactions between positive and negative regions with multi-scales. In the expansion region, the momentum transports are mainly dominated by the streamwise large-scale structures and rare small-scale motions between high and low speed flow can be observed. A quantitative evident is further provided in Figure $10(c, d)$, where the PDFs of streamwise vorticity at $y_{n} / \delta_{\text {ref }}=0.015$ and 0.24 are present. An increase of the expansion angle produces a smaller streamwise vorticity in the near wall region, but no considerable changes can be observed in the outer layer, supporting that near-wall small-scale turbulent structures are dramatically quenched after the expansion corner.

Figure 11 shows contours of the temporal cross-correlation coefficient between the streamwise velocity fluctuations and $\tau_{x}^{\prime}$ for $\beta=0^{0}$ and $\beta=10^{0}$, respectively. It is defined as [31]

$$
C_{u^{\prime} \tau^{\prime} x}\left(y_{n}, \Delta t\right)=\frac{\overline{u^{\prime}\left(x_{0}, y_{n}, t\right) \tau_{x}^{\prime}\left(x_{0}, 0, t+\Delta t\right)}}{u_{\mathrm{rms}} \tau_{x, \mathrm{rms}}}
$$

Here, the time signal of $\tau_{x}^{\prime}$ is taken at $x_{0}=30$ and the streamwise velocity probe is directly located above the WSS probe along the wall-normal direction. For $\beta=0^{0}$, it is seen that significant levels of correlation occurs in the near-wall region $\left(y_{n} / \delta_{\text {ref }}<0.1\right)$. Its magnitude decreases rapidly with an increased positive time lag as the velocity probe moves away from the wall. This is related to the large scale structures inclined with respect to the wall in the streamwise direction. As shown in Figure 11(b), the general distribution is similar to that of the flat-plate; however, a significant augmentation of correlation in the outer layer is clearly highlighted in the figure. Even at $y_{n} / \delta_{\text {ref }} \approx 0.2$, the velocity fluctuation still strongly correlate with the fluctuating WSS. Recalling the analysis of coherent structures in Figure 10, it is inferred that since the two-point cross-correlation map is actually 


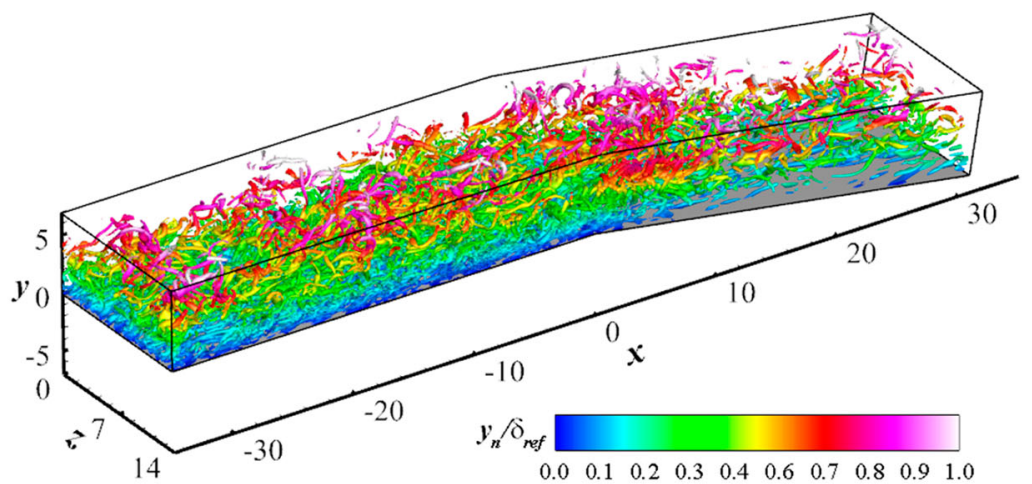

(a)

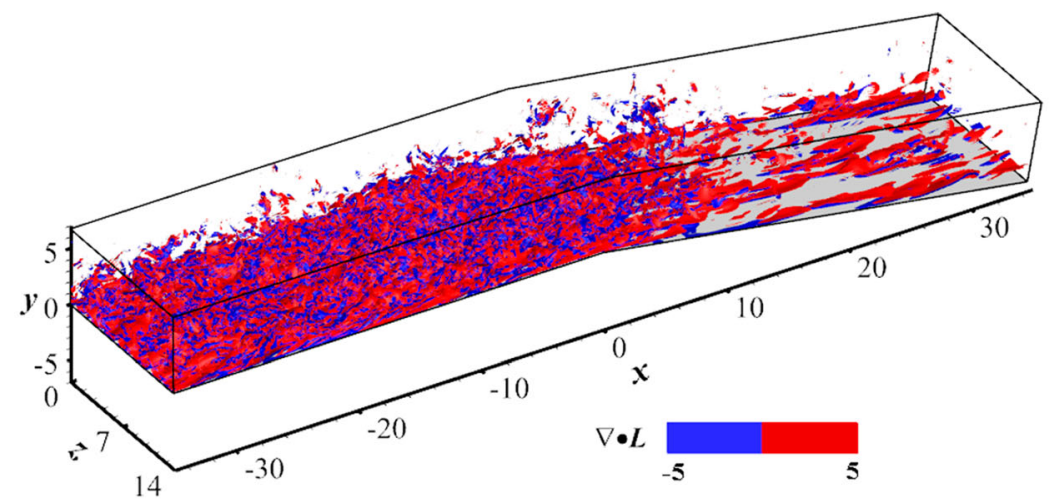

(b)

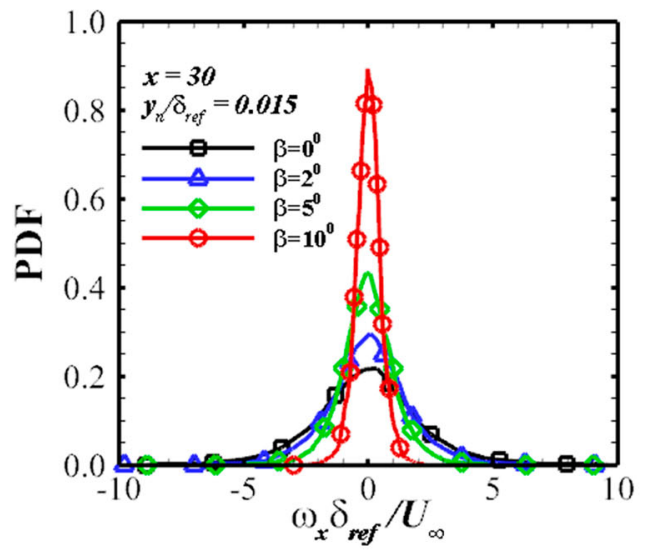

(c)

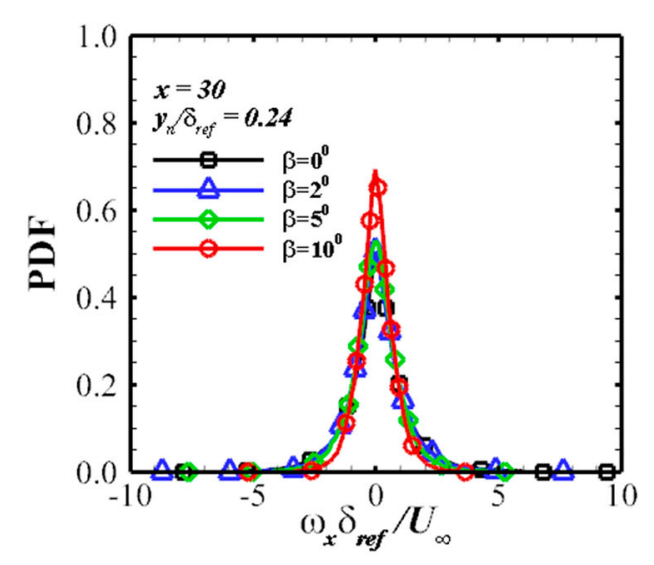

(d)

Figure 10. (a) Iso-surface of $Q$ criterion at $Q / Q_{\max }=0.01$ coloured by the wall-normal distance $y_{n} / \delta_{\text {ref }}$ for $\beta=10^{0}$; (b) momentum transport visualised by iso-surfaces of Lamb vector divergence $\nabla \cdot \boldsymbol{L}$ for $\beta=10^{0} ;(c, d)$ PDFs of streamwise vorticity at two wall-normal locations. 


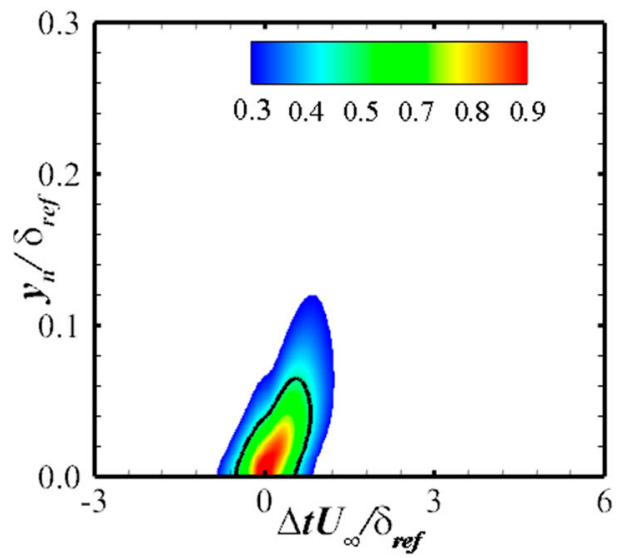

(a)

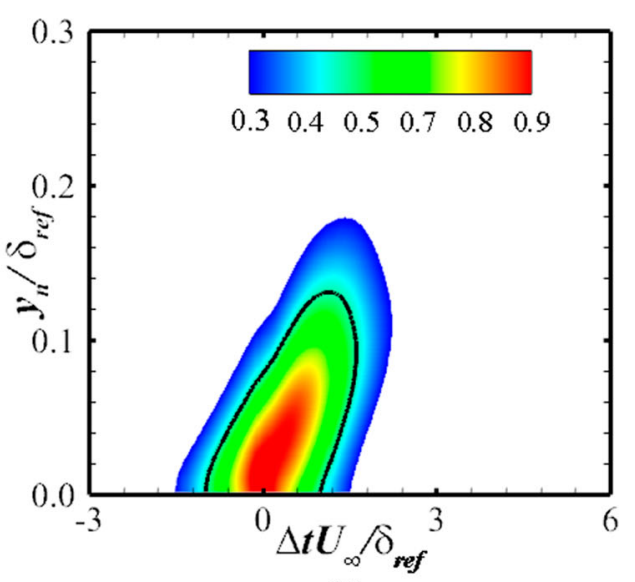

(b)

Figure 11. Contours of the temporal cross-correlation between the streamwise velocity fluctuation and $\tau_{x}^{\prime}$ at $x=30$ : (a) $\beta=0^{0}$; (b) $\beta=10^{0}$. The black line denotes the correlation value of 0.5 .

determined by the joint contribution from both the small-scale and large-scale coherent motions; the reduction of the relative weighting of the former and the amplification of the correlation with the latter naturally biases the correlation map towards the latter.

\section{Statistical analysis of the WSS}

The root-mean square (r.m.s) of the WSS fluctuations is quantitatively compared in Figure 12. According to the empirical correlations proposed by Orlu \& Schlatter [32] and Dianel et al. [23], the r.m.s levels satisfy a logarithmic dependence on the Reynolds number $R e_{\tau}$ by the following equations:

$$
\tau_{x, \mathrm{rms}} / \tau_{x, \mathrm{av}}=0.298+0.018 \ln \mathrm{Re}_{\tau} \quad \text { and } \quad \tau_{z, \mathrm{rms}} / \tau_{x, \mathrm{av}}=0.164+0.018 \ln \mathrm{Re}_{\tau},
$$

where the only difference between the two components is a constant displacement. The Reynolds number $R e_{\tau}$ for the flat-plate at $x=30$ is estimated to be nearly 300 . The corresponding r.m.s values for $\tau_{x}^{\prime}$ and $\tau_{z}^{\prime}$ are calculated to be approximately 0.4 and 0.29 , respectively, in good agreement with the theoretical solutions. It is found that the r.m.s value undergoes a sharp drop and attains a minimum in the vicinity of corner, followed by a slow increase in the recovery region. The reduction is significantly enhanced as the deflection angle $\beta$ is increased. With respect to $\beta=0^{0}$, the maximum decreases of both components for $\beta=10^{0}$ are approximately $66 \%$ and $75 \%$, respectively. The figure also highlights substantially different recovery process for $\tau_{x}^{\prime}$ and $\tau_{z}^{\prime}$. Obviously, the streamwise WSS fluctuation experiences a rapid recovery, but the recovery of the spanwise component is relatively slower. In particular, the spanwise component seems to be almost unchanged for $\beta=10^{0}$ beyond $x=10$, in contrast to a consistent increase is observed in other three cases. This can be explained as that the absolute r.m.s value of the spanwise component for $\beta=10^{0}$ exhibits a continuous decrease in the recovery region, while $\tau_{z \text {,rms }}$ nearly keeps constant for smaller deflection angles. Since $\tau_{x \text {,av }}$ gradually decreases as the boundary layer develops along the expansion corner for all cases, the reason accounting for the discrepancy 


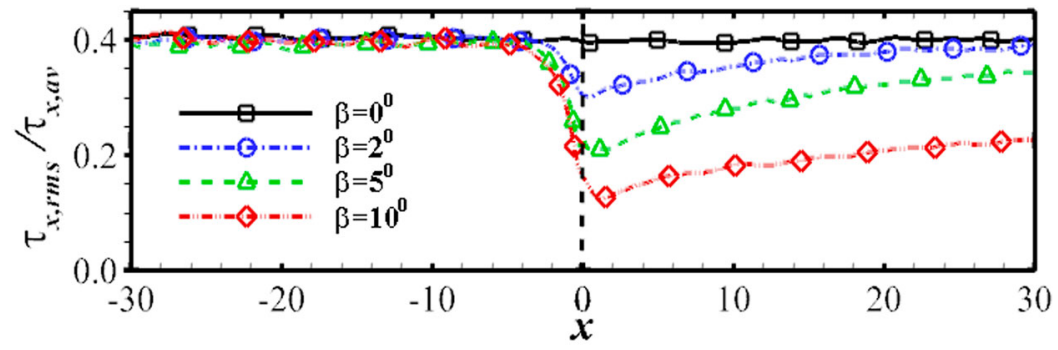

(a)

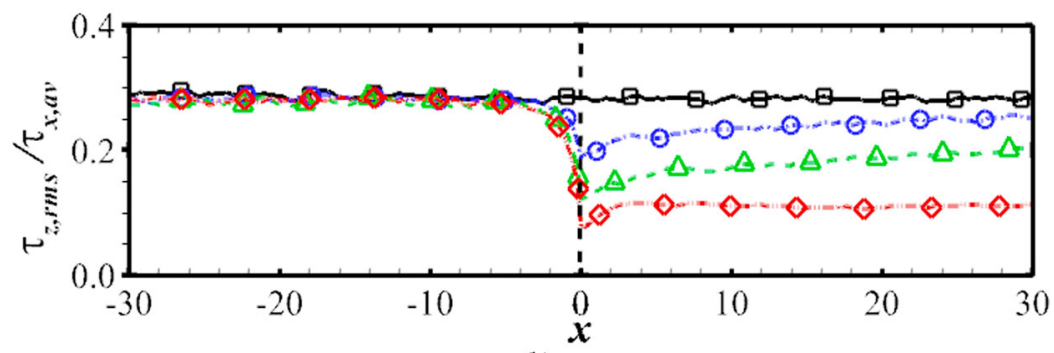

(b)

Figure 12. Distributions of the normalised r.m.s values of the WSS: (a) $\tau_{x, r m s} / \tau_{x, a v}$; (b) $\tau_{z, r m s} / \tau_{x, a v}$.

becomes apparent. Notably, the fluctuating WSS is not fully recovered to its equilibrium state due to the short length of recovery region used in the present simulation. In fact, Sun et al. [11] pointed out that it may take at least ten times of the incoming TBL thickness for the boundary layer to recover downstream of the expansion.

Figure 13(a) and Figure 14(a) shows the PDFs of both components of the WSS normalised by its mean value, where the log-normal and Gaussian distributions are also plotted, respectively. Only the statistics at $x=30$ (after the expansion corner) are shown. Here, a logarithm scale is used in this figure to emphasise the tail region and some interesting features deserve to be highlighted. For $\beta=0^{0}$, the PDF of $\tau_{x} / \tau_{x \text {,av }}$ is positively skewed and characterised by the log-normal distribution. It is worth pointing out that the negative tail of the PDF distribution labelled by the shade region further confirms the occurrence of the negative streamwise shear stress, associated with the rare backflow extreme events, as found in DNS simulations of channel flow [33] and boundary layer [23] with zero pressure gradient. In contrast, the PDF of $\tau_{z} / \tau_{z \text {,av }}$ is nearly symmetric and the peak is much higher than that of the Gaussian distribution. Moreover, the tails in the PDF distributions for both components are significantly narrowed with increasing deflection angle $\beta$, implying the decrease of the occurrence probability of negative and positive extreme events. It is interesting to note that the PDF distribution of the streamwise component for $\beta=10^{0}$ is only dominated by positive tails and a higher peak around the maximum of the PDF, suggesting that no flow reversals occur in the expansion region. Furthermore, the PDFs of the normalised fluctuations are also shown in Figure 13(b) and Figure 14(b) to investigate the expansion effect on the variance. It is found that the positively skewed PDF profiles of $\tau_{x}^{\prime} / \tau_{x, \mathrm{rms}}$ at various $\beta$ fail to collapse with each other; namely, the negative part presents a more distinct dependency on $\beta$, resulting in the reduction of the spreading of the PDF 


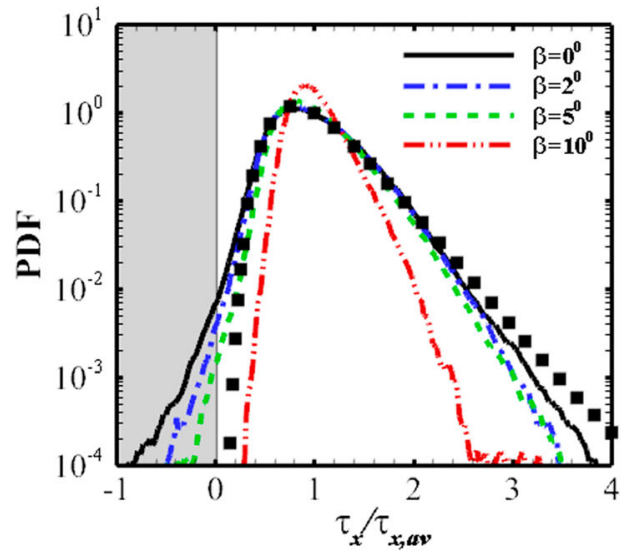

(a)

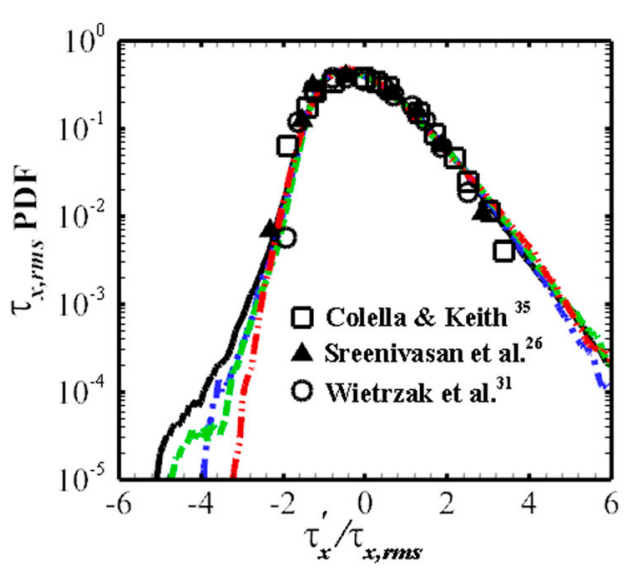

(b)

Figure 13. PDFs of the normalised streamwise WSS and its fluctuation at $x=30$ : (a) $\boldsymbol{\tau}_{x} / \boldsymbol{\tau}_{\boldsymbol{x}, \boldsymbol{a}}$; (b) $\boldsymbol{\tau}^{\prime}{ }_{x} / \boldsymbol{\tau}_{\boldsymbol{x}, \boldsymbol{r m s}}$. Solid squares denote the log-normal distribution[35].

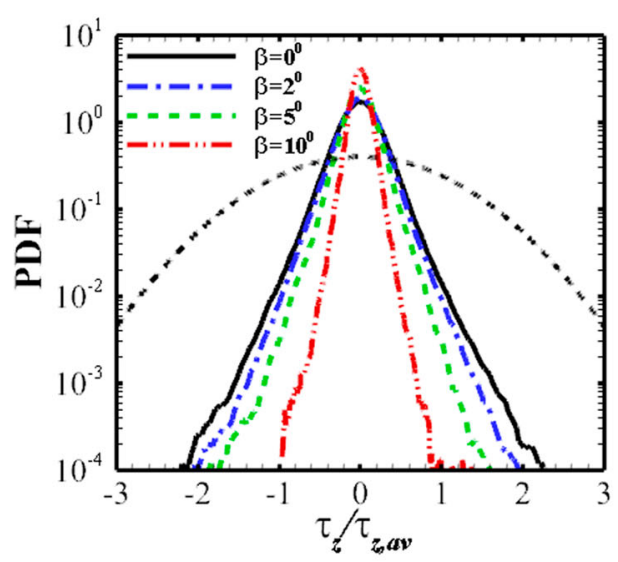

(a)

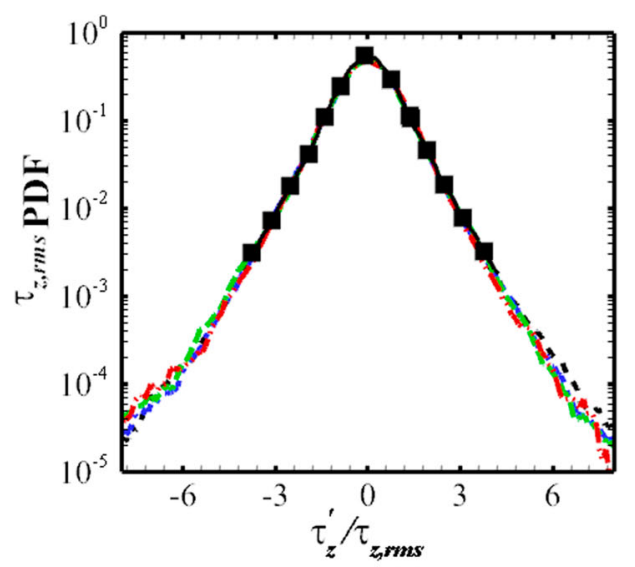

(b)

Figure 14. PDFs of the normalised spanwise WSS and its fluctuation at $x=30$ : (a) $\boldsymbol{\tau}_{z} / \boldsymbol{\tau}_{z, a v}$; (b) $\boldsymbol{\tau}^{\prime}{ }_{z} / \boldsymbol{\tau}_{z, r m s}$. Dashed line denotes the log-normal distribution and solid squares denotes the data of Daniel et al. [23].

$\left(\tau_{x}^{\prime} / \tau_{x, \text { rms }}\right)$ profile with the increase of $\beta$. However, a quasi-symmetric PDF distribution that is invariant to $\beta$ is observed for the normalised $\tau_{z}^{\prime} / \tau_{z, \text { rms }}$.

The PDFs of the yaw angle $\psi_{\tau}$ of the shear stress vector $\vec{\tau}\left(\tau_{x}, \tau_{z}\right)$, defined as $\psi_{\tau}=\arctan \left(\tau_{z} / \tau_{x}\right)$, are plotted as a function of $\beta$ in Figure 15 together with the Gaussian distribution for reference. In DNS of the wall-bounded flow, Jeon et al. [34] and Daniel et al. [23] suggested that the yaw angle of instantaneous wall shear stress events is mainly concentrated between $-45^{0}$ and $45^{0}$ with respect to the streamwise direction. The experiments of Grosse \& Schroder [23] further reported the angle up to $40^{\circ}$. As we can see, $\psi_{\tau}$ is mainly concentrated within $\pm 45^{0}$ for $\beta=0^{0}, 2^{0}$ and $5^{0}$. However, as $\beta$ increases to $10^{0}$, the PDF profile becomes remarkably narrowed, and an overshot appears in the peak value. This observation, to our understanding, infers the dramatical reduction of the spanwise 


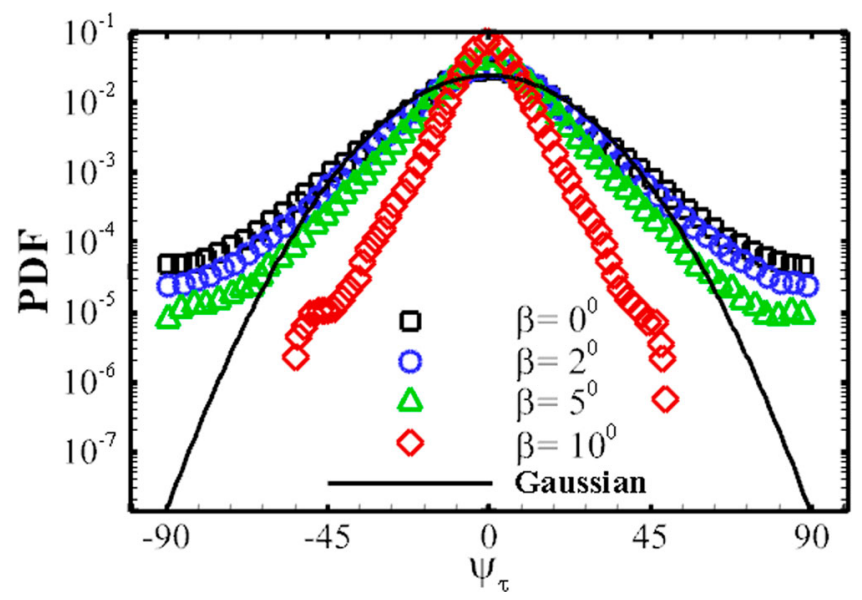

Figure 15. PDF profiles of the yaw angle $\psi_{\tau}$ of the WSS vector $\overrightarrow{\boldsymbol{\tau}}\left(\boldsymbol{\tau}_{\boldsymbol{x}}, \boldsymbol{\tau}_{z}\right)$ at $x=30$.

meandering of the streaky structures in the field of WSS, which in turn can be interpreted as the suppression of the intermittency in the relaminarization process.

The expansion effect on the WSS vector $\vec{\tau}$ is further addressed in Figure 16(a-c) by comparing the joint PDFs of $\left(\tau_{x}^{\prime} / \tau_{x, \text { av }}, \tau_{z}^{\prime} / \tau_{x \text {,av }}\right)$ of the expanded cases to that of the flat-plate case (with $\beta=0^{0}$ ). While Figure 16(d-f) show the corresponding joint PDFs between the length of instantaneous WSS vectors $(|\tau|)$ and their yaw angles $\left(\psi_{\tau}\right)$. Only the statistics at $x=30$ are shown in Figure 16. Note that the contours of Joint PDFs are normalised by their own maximum values, while $\tau_{x}^{\prime}$, $\tau_{z}^{\prime}$ and $|\tau|$ are normalised by the mean WSS $\left(\tau_{x, \mathrm{av}}\right)$, instead of by the RMS value $\left(\tau_{x, r m s}\right.$ or $\left.\tau_{z, r m s}\right)$ used in Figures 13 and 14, to highlight the variation of the fluctuation level. The maps of Joint PDF $\left(\tau_{x}^{\prime} / \tau_{x, a v}, \tau_{z}^{\prime} / \tau_{x, a v}\right)$ are seen to bias towards the $+\tau_{x}^{\prime}$ direction but are symmetric about $\tau_{x}^{\prime}=0$, consistent with the univariate PDF profiles shown in Figure 13. The larger $\beta$ the more contraction of the extent of Joint PDF $\left(\tau_{x}^{\prime} / \tau_{x, a v}, \tau_{z}^{\prime} / \tau_{x, a v}\right)$, suggesting that the level of the reduction of the fluctuating intensity of WSS is proportional to the deflection angle. Nevertheless, Joint $\operatorname{PDF}\left(|\tau| / \tau_{x, a v}\right.$, $\psi_{\tau}$ ) presents a remarkable shrinking only when $\beta=10^{0}$. Recalling that the relatively low probability of extreme $\psi_{\tau}$ events evidences the dominating weight of $\tau_{x}^{\prime}$ over $\tau_{z}^{\prime}$, this observation infers that the relaminarization process, which is the most prominent for $\beta=10^{0}$, is characterised as the regularisation of the wavy motion of streaky-liked WSS structures.

Finally, the pre-multiplied energy spectra of the fluctuating WSS at $x=30$ are given in Figure 17. The spectra are computed from the time-series signals of $\tau_{x}^{\prime}(t)$ or $\tau_{z}^{\prime}(t)$ at a fixed $(x, z)$ position with a time duration of $250 \delta_{\text {ref }} / U_{\infty}$. To enlarge the ensemble size for statistical convergence, the time-signals cross the whole spanwise span are used, each of them are split into 6 segments with 50\% overlap, whose Welch's power spectral densities are calculated and then averaged among all ensembles. For a better visualisation, the $x$ axis in the spectra of $\tau_{z}^{\prime}$ is right-shifted by multiplying the frequencies by a factor of 10 . As shown in Figure 17, the normalised peak frequency of $\Phi_{\tau_{x} \tau_{x}}$ appears at $\omega v_{w} / u_{\tau}^{2}=0.1$ 0.2 , and is smaller than that of the spanwise component which locates at $\omega \nu_{w} / u_{\tau}{ }^{2}=0.3$. This is consistent with the visualisation in Figure 8 that the length scale of $\tau_{x}^{\prime}$ structures is distinctly larger than that of $\tau_{z}^{\prime}$ structures. For $\tau_{x}^{\prime}$, the energy content around the peak 


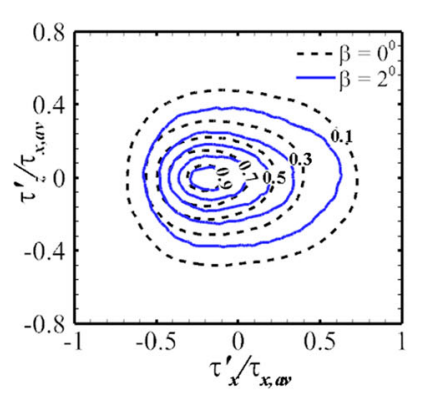

(a)

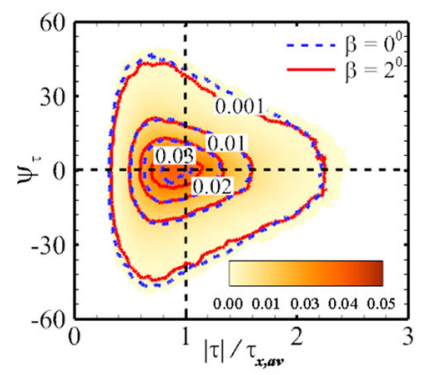

(d)

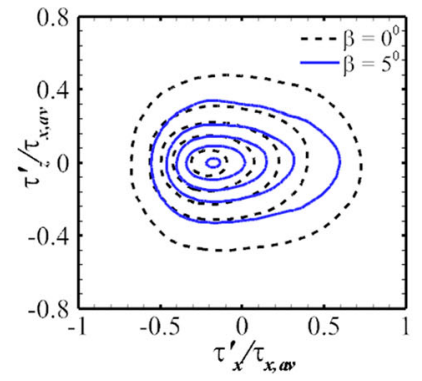

(b)

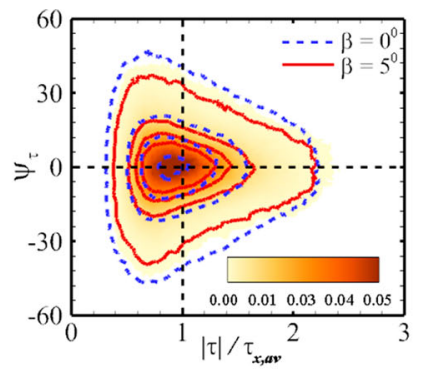

(e)

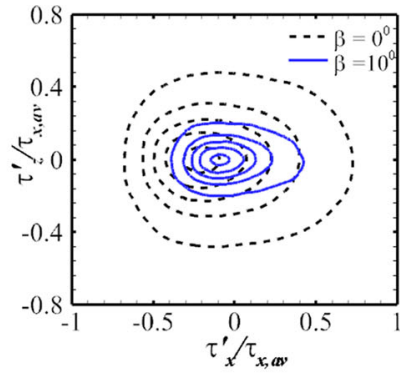

(c)

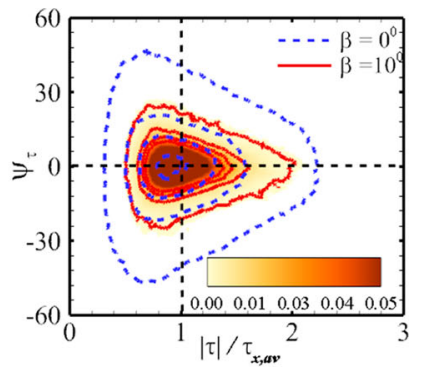

(f)

Figure 16. $(a, b, c)$ Joint PDFs between the streamwise and spanwise components of the normalizd fluctuating WSS; $(d, e, f)$ joint PDFs of the magnitude and yaw angle of instantaneous WSS vectors.

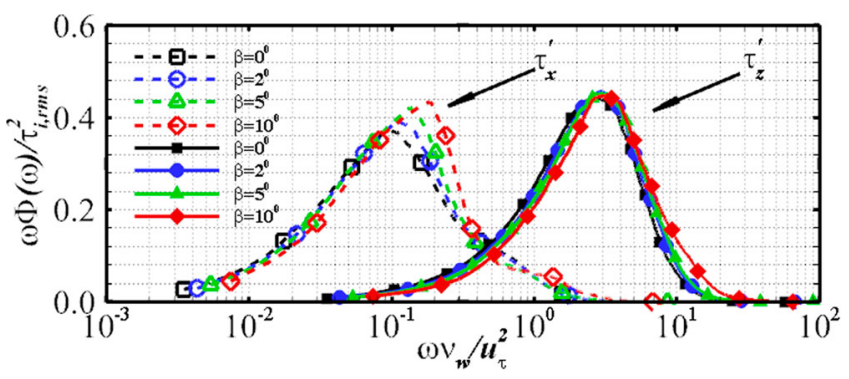

Figure 17. Pre-multiplied energy spectra of WSS fluctuations taken at $x=30$.

frequency in $\Phi_{\tau_{x} \tau_{x}}$ monotonically increases with $\beta$, so does the magnitude of the peak frequency. Combing with the observation from Figure 9 that the streamwise length of $\tau_{x}^{\prime}$ structures increases with $\beta$, a higher convection speed of $\tau_{x}^{\prime}$, i.e. $U_{c, \tau_{x}}$, can be expected in the case of larger $\beta$. A detailed investigation via two-point time-lag correlation shows that $U_{\mathcal{c}, \tau_{x}}=0.5-0.6 U_{\infty}$ at $\beta=0^{\circ}$ while increases to $U_{\mathcal{c}, \tau_{x}}=0.6-0.8 U_{\infty}$ at $\beta=10^{\circ}$. This is also consistent with the finding in Figure 13(b) that the possibility of extreme $\tau_{x}^{\prime}$ events with negative sign slightly decreases with the increase of $\beta$, since these negative events convect downstream at lower speed. 


\section{Conclusions}

Direct numerical simulation of a supersonic TBL over an expansion corner has been performed to investigate the effect of abrupt expansion on the characteristics of the fluctuating WSS. Three deflection angles, corresponding to small $\left(\beta=2^{0}, 5^{0}\right)$ or large $\left(\beta=10^{0}\right)$ expansion, are considered. Streaky structures of both the streamwise and spanwise components of the fluctuating WSS are seen to be significantly weakened shortly after the expansion corner but experience a different regrowth in the far downstream. Crosscorrelation analysis shows that these regrowing WSS streaks have comparably larger length scales due to the weakening of small-scale random fluctuations and the amplified correlation with large-scale motions. The increase of the length scale is proportional to the deflection angle. Moreover, a prominent alignment of the instantaneous WSS vector along the streamwise direction is evident for the largest expansion case (with $\beta=10^{0}$ ), this suggests the suppression of the spanwise meandering of WSS streaks and the reduction of the intermittency in the relaminarization process. Spectral analysis shows that the characteristic time scale of the spanwise component of WSS is less affected by the expansion effect, but that of the streamwise component decreases with the increase of the reflection angle, leading to a distinct increase of its convection speed in large expansion case.

\section{Acknowledgements}

We are deeply grateful to Dr. Chong Pan from Beihang University for his fruitful discussion and help in improving the quality of this paper.

\section{Disclosure statement}

No potential conflict of interest was reported by the author(s).

\section{Funding}

This work was supported by the National Key Research and Development Program of China Natural Science Foundation of China Key Research and Development Program of China (2019YFA0405300, 2016YFA0401200), NSFC Projects (11972356, 91852203), National Numerical Windtunnel project, Science Challenge Project (TZ2016001), and Strategic Priority Research Program of Chinese Academy of Sciences (XDC01000000).

\section{ORCID}

Xinliang $L i$ (D) http://orcid.org/0000-0002-4264-9620

\section{References}

[1] Sun MB, Zhong Z, Liang JH, et al. Experimental investigation on combustion performance of cavity-strut injection of supercritical kerosene in supersonic model combustor. Acta Astronaut. 2016;127:112-119.

[2] Narasimha R, Sreenivasan KR. Relaminarization in highly accelerated turbulent boundary layers. J Fluid Mech. 1973;61(3):417-447.

[3] Dussauge JP, Gaviglio J. The rapid expansion of a supersonic turbulent flow: role of bulk dilatation. J Fluid Mech. 1987;174:81-112.

[4] Smith DR, Smits AJ. The rapid expansion of a turbulent boundary layer in a supersonic flow. Theor Computat Fluid Dyn. 1991;2:319-328. 
[5] Arnette SA, Samimy M, Elliott GS. Structure of supersonic turbulent boundary layer after expansion regions. AIAA J. 1995;33(3):430-438.

[6] Arnette SA, Samimy M, Elliott GS. The effects of expansion on the turbulence structure of compressible boundary layers. J Fluid Mech. 1998;367:67-105.

[7] Chung KM, Lu FK. Damping of surface pressure fluctuations in hypersonic turbulent flow past expansion corners. AIAA J. 1993;31(7):1229-1234.

[8] Dawson JA, Samimy M, Arnette SA. Effects of expansions on a supersonic boundary layer: surface pressure measurements. AIAA J. 1994;32(11):2169-2177.

[9] Nguyen T, Behr M, Reinartz B. "Numerical investigation of compressible turbulent boundary layer over expansion corner. American Institute of Aeronautics and Astronautics. 2009. AIAA Paper 2009-7371.

[10] Teramoto S, Sanada H, Okamoto K. Dilatation effect in relaminarization of an accelerating supersonic turbulent boundary layer. AIAA J. 2017;55(4):1469-1474.

[11] Sun MB, Hu ZW, Sandham ND. Recovery of a supersonic turbulent boundary layer after an expansion corner. Phys Fluids. 2017;29:076103.

[12] Bookey P, Wyckham C, Smits A. New experimental data of STBLI at DNS/LES accessible Reynolds numbers, AIAA paper No. 2005-309, 2005.

[13] Wu M, Martin MP. Direct numerical simulation of supersonic turbulent boundary layer over a compression ramp. AIAA J. 2007;45(4):879-889.

[14] Narasimha R, Viswanath PR. Reverse transition at an expansion corner in supersonic flow. AIAA J. 1975;13(5):693-695.

[15] Tong FL, Li XL, Duan YH, et al. Direct numerical simulation of supersonic turbulent boundary layer subjected to a curved compression ramp. Phys Fluids. 2017;29:125101.

[16] Martin MP, Taylor EM, Wu M, et al. A bandwidth-optimized WENO scheme for the effective direct numerical simulation of compressible turbulence. J Comput Phys. 2006;220.

[17] Pirozzoli S, Grasso F, Gatski TB. Direct numerical simulation and analysis of a spatially evolving supersonic turbulent boundary layer at $M=2.25$. Physics of Fluids. 2004;16:530-545.

[18] Schlatter P, Orlu R. Assessment of direct numerical simulation data of turbulent boundary layers. J Fluid Mech. 2010;659:116-126.

[19] Pirozzoli S, Bernardini M, Grasso F. Direct numerical simulation of transonic shock/boundary layer interaction under conditions of incipient separation. J Fluid Mech. 2010;657: 361-393.

[20] Erm LP, Joubert J. Low Reynolds number turbulent boundary layers. J Fluid Mech. 1991;230:1-44.

[21] Wu X, Moin P. Direct numerical simulation of turbulence in a nominally zero-pressuregradient flat-plate boundary layer. J Fluid Mech. 2009;630:5-41.

[22] Hu ZW, Morfey CL, Sandham ND. Wall pressure and shear stress spectra from direct numerical simulations of channel flow up to $R e_{\tau}=1440$. AIAA Journal. 2006;44(7):1541-1549.

[23] Daniel CD, Laizet S, Vassilicos JC. Wall shear stress fluctuations: mixed scaling and their effects on velocity fluctuations in a turbulent boundary layer. Physics of Fluids. 2017;29:055102.

[24] Grosse S, Schröder W. Wall-shear stress patterns of coherent structures in turbulent duct flow. J Fluid Mech. 2009;633:147-158.

[25] Nottebrock B, Geurts KJ, Schröder W. "Wall-shear stress measurements in an adverse pressure gradient turbulent boundary layer. 2014: 2014-2098. AIAA Paper.

[26] Sreenivasan KR, Antonia RA. Properties of wall-shear stress fluctuations in turbulent duct flow. J Appl Mech. 1977;44:389-395.

[27] Pan C, Kwon Y. Extremely high wall-shear stress events in a turbulent boundary layer. J Phys Conf. Series. 2018;1001:012004.

[28] Bernardini M, Pirozzoli S. "Wall pressure fluctuations beneath supersonic turbulent boundary layers. Phys Fluids. 2011;23:085102.

[29] Jeong J, Hussain F. On the identification of a vortex. J Fluid Mech. 1995;285:69-94.

[30] Hamman CW, Klewicki JC, Kirby RW. On the Lamb vector divergence in Navier-Stokes flows. J Fluid Mech. 2008;610:261-284. 
[31] Wietrzak A, Lueptow RM. Wall shear stress and velocity in a turbulent axisymmetric boundary layer. J Fluid Mech. 1994;259:191-218.

[32] Orlu R, Schlatter P. On the fluctuating wall-shear stress in zero pressure-gradient turbulent boundary layer flows. Phys Fluids. 2011;23:021704.

[33] Lenaers P, Li Q, Brethouwer G, et al. Rare backflow and extreme wall-normal velocity fluctuations in near-wall turbulence. Phys Fluids. 2012;24:035110.

[34] Jeon S, Choi H, Yoo JY, et al. Space-time characteristics of the wall shear-stress fluctuations in a low-Reynolds-number channel flow. Phys Fluids. 1999;11:3084-3094.

[35] Colella KJ, Keith WL. Measurements and scaling of wall shear stress fluctuations. Exp Fluids. 2003;34:253-260. 